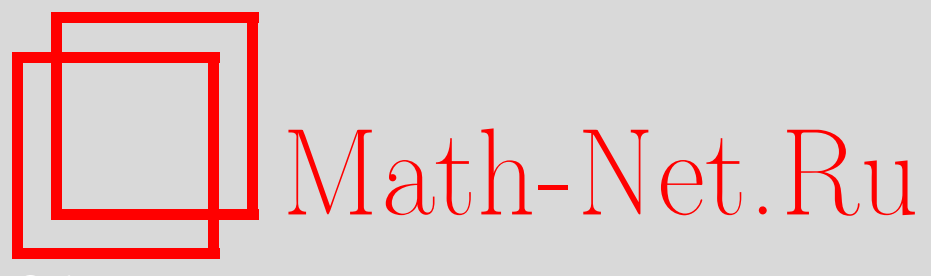

А. В. Кудинов, И. Б. Шапировский, О финитной аппроксимируемости модальных логик конечной глубины, УМH, 2016, том 71, выпуск 1, 175-176

DOI: https://doi.org/10.4213/rm9686

Использование Общероссийского математического портала Math-Net.Ru подразумевает, что вы прочитали и согласны с пользовательским соглашением http://www.mathnet.ru/rus/agreement

Параметры загрузки:

IP : 34.227 .88 .159

26 апреля 2023 г., 08:29:39

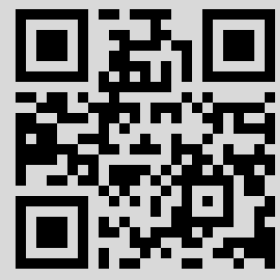




\section{О финитной аппроксимируемости модальных логик конечной глубины}

\section{А. В. Кудинов, И. Б. Шапировский}

В настоящей заметке рассматриваются пропозиционалъные нормалъные модалъные логики [2]. Финитная аппроксимируемость модальной логики означает ее полноту относительно некоторого класса конечных шкал. В случае конечно аксиоматизируемой логики это свойство немедленно влечет ее разрешимость (теорема Харропа). Вопрос о финитной аппроксимируемости модальных логик изучен достаточно хорошо, это свойство доказано для многих логик (см., например, [2], [1]). Логики без финитной аппроксимируемости тоже известны, хотя в одномодальном случае они скорее экзотика и обычно строятся искусственно. Имеется еще один случай - логики, для которых вопрос о финитной аппроксимируемости открыт. По-видимому, самым известным таким примером является логика $\mathrm{K}_{3}^{2}$, задаваемая единственной простой формулой $\square \square p \rightarrow \square \square \square p$. Вообще, финитная аппроксимируемость и даже разрешимость логик $\mathrm{K}_{n}^{m}$, задаваемых аксиомами $\square^{m} p \rightarrow \square^{n} p$, является старой открытой проблемой (см. [2; проблема 11.2], [8; проблема 6]): ответ не известен ни для каких $m, n>1$, $m \neq n$.

При $n>m$ в логике $\mathrm{K}_{n}^{m}$ вырази́м модальный оператор, соответствующий транзитивному рефлексивному замыканию бинарного отношения. Мы будем называть такие логики предтранзитивными. Формально L предтранзитивна, если существует формула $\chi(p)$ такая, что для любой модели $M$ такой, что $M \vDash \mathrm{L}$, и для любой точки этой модели имеет место эквивалентность $M, w \vDash \chi(p) \Longleftrightarrow \forall u\left(w R^{*} u \Rightarrow M, u \vDash p\right)$, где $R^{*}-$ транзитивное рефлексивное замыкание отношения $R$ модели $M$.

Предтранзитивность логики эквивалентна тому, что для некоторого $k \geqslant 0$ она содержит формулу $k$-транзитивности $\square^{\leqslant k} p \rightarrow \square^{k+1} p$, где $\square^{\leqslant k} \varphi=\bigwedge_{i=0}^{k} \square^{i} \varphi$ (см. [3]). В частности, при $n>m$ логика $\mathrm{K}_{n}^{m}$ является $(n-1)$-транзитивной. Логику, заданную единственной аксиомой $\square^{\leqslant m} p \rightarrow \square^{m+1} p$, будем обозначать $\mathrm{K}_{\leqslant m}$. Таким образом, $\mathrm{K}_{\leqslant m}$ - минимальная $m$-транзитивная логика. При $m>1$ финитная аппроксимируемость $\mathrm{K}_{\leqslant m}$ также неизвестна.

Для полной по Крипке логики L ее финитная аппроксимируемость означает следующее: если формула $\varphi$ выполнима в L-шкале, то она выполнима в конечной L-шкале. Из теоремы Салквиста следует, что логики $\mathrm{K}_{n}^{m}, \mathrm{~K}_{\leqslant m}$ полны по Крипке; класс шкал каждой из этих логик характеризуется достаточно простым условием первого порядка. Так, $\mathrm{K}_{3}^{2}$-шкалы характеризуются условием $R \circ R \circ R \subseteq R \circ R$, где ०композиция отношений; и вообще, $\mathrm{K}_{n}^{m}$-шкалы характеризуются условием $R^{n} \subseteq R^{m}$, a $\mathrm{K}_{\leqslant m}$-шкалы - условием $R^{m+1} \subseteq \bigcup_{i \leqslant m} R^{i}$. Таким образом, неизвестно, существует ли преобразование бесконечных шкал в конечные, при котором бы сохранялась выполнимость данной формулы и одновременно указанные условия. Нам удалось описать такое преобразование в случае, когда глубина шкалы (т. е. наибольшая из мощностей цепей в частичном порядке, индуцированном отношением $R$ ) конечна.

Лемма 1. Пусть L - одна из логик $\mathrm{K}_{n}^{m}, \mathrm{~K}_{\leqslant m}, n>m \geqslant 1$. Если формула выполнима в L-шкале конечной глубины, то она выполнима в конечной L-шкале той же глубины.

Известно [2], что ограничение на глубину предпорядка можно выразить модальными формулами. Определим аналог этих формул для произвольной предтранзитивной L. Положим $\square^{*} \varphi=\square^{\leqslant k} \varphi$ для наименьшего $k$ такого, что $\mathrm{K}_{\leqslant k} \subseteq \mathrm{L}$. В частности, $\square^{*} \varphi=\square^{\leqslant n-1} \varphi$ для $\mathrm{K}_{n}^{m}, n>m$. Также положим $\diamond^{*} \varphi=\neg \square^{*} \neg \varphi$. Формулы $B_{h}$ определяются по индукции: $B_{1}=p_{1} \rightarrow \square^{*} \diamond^{*} p_{1}, B_{h+1}=p_{h+1} \rightarrow \square^{*}\left(\diamond^{*} p_{h+1} \vee B_{h}\right)$.

Исследование выполнено в ИППИ РАН за счет гранта Российского научного фонда (проект № 14-50-00150).

DOI: $10.4213 / \mathrm{rm} 9686$ 
ПреДЛОЖенИЕ 2. Для предтранзитивной логики L $u$ L-шкалы $F$ имеет место эквивалентность: $F \vDash B_{h} \Longleftrightarrow$ глубина $F$ не превосходит $h$.

Расширение L формулой $B_{h}$ обозначим L. $B_{h}$.

Теорема 3. Пусть L - предтранзитивная логика. Тогда: 1) L. $B_{1} \supseteq$ L. $B_{2} \supseteq$ L. $\left.B_{3} \supseteq \cdots \supseteq \mathrm{L} ; 2\right)$ если L непротиворечива, то все L. $B_{h}, h \geqslant 1$, непротиворечивы; 3) если L каноническая, то все L. $B_{h}, h \geqslant 1$, канонические.

СледствиЕ 4 . Для всех $n>m \geqslant 1, h \geqslant 1$ логики $\mathrm{K}_{n}^{m} . B_{h}, \mathrm{~K}_{\leqslant m} . B_{h}$ канонические и, следовательно, полные по Крипке.

В общем случае предтранзитивные логики конечной глубины устроены существенно сложнее, чем соответствующие расширения логики предпорядков S4. Так, все логики $\mathrm{S} 4 . B_{h}$ локально табличны, логика $\mathrm{S} 4 . B_{1}=\mathrm{S} 5$ предтаблична, однако ни локальная табличность, ни предтабличность не имеют места даже в "простейшем" случае $\mathrm{K}_{\leqslant 2} . B_{1}$ [7], [5]. Это же верно для всех логик $\mathrm{K}_{n}^{m} . B_{h}, \mathrm{~K}_{\leqslant m} . B_{h}(n>m \geqslant 2)$, поскольку они включены в $\mathrm{K}_{\leqslant 2} . B_{1}$. Тем не менее эти логики оказываются финитно аппроксимируемыми. Уже в случае высоты 1 доказательство этого факта достаточно нетривиально (особенно для логик $\mathrm{K}_{n}^{m} . B_{1}$ при $n>m+1$ ); финитная аппроксимируемость логик $\mathrm{K}_{\leqslant m} . B_{1}$ была доказана в [4], предтранзитивных логик $\mathrm{K}_{n}^{m} \cdot B_{1}-$ в [6]. Следующая теорема распространяет эти результаты на случай произвольной конечной глубины.

Теорема 5. Для всех $n>m \geqslant 1, h \geqslant 1$ логики $\mathrm{K}_{n}^{m} \cdot B_{h}, \mathrm{~K}_{\leqslant m} \cdot B_{h}$ финитно аппроксимируемы.

Одним из следствий этой теоремы является критерий финитной аппроксимируемости логик $\mathrm{K}_{n}^{m}, \mathrm{~K}_{\leqslant m}$.

СледСтвие 6. Пусть L - одна из логик $\mathrm{K}_{n}^{m}, \mathrm{~K}_{\leqslant m}, n>m \geqslant 1$. Тогда

L финитно аппроксимируема $\Longleftrightarrow \mathrm{L}=\bigcap_{h \geqslant 1} \mathrm{~L} \cdot B_{h}$.

\section{Список литературы}

[1] P. Blackburn, M. de Rijke, Y. Venema, Modal logic, Cambridge Univ. Press, Cambridge, 2001. [2] A. Chagrov, M. Zakharyaschev, Modal logic, Oxford Univ. Press, New York, 1997. [3] D. M. Gabbay, V. Shehtman, D. Skvortsov, Quantification in nonclassical logic, v. 1, Elsevier, Amsterdam, 2009. [4] R. Jansana, Notre Dame J. Formal Logic, 35:1 (1994), 88-98. [5] Z. Kostrzycka, MLQ Math. Log. Q., 54:6 (2008), 617-624. [6] A. Kudinov, I. Shapirovsky, "Finite model property of pretransitive analogs of S5", Topology, algebra and categories in logic (Marseille, France, 2011), 2011, 261-264. [7] Y. Miyazaki, Advances in modal logic, v. 5, King's Coll. Publ., London, 2005, 171-190. [8] F. Wolter, M. Zakharyaschev, Handbook of modal logic, Stud. Log. Pract. Reason., 3, Elsevier, Amsterdam, 2007, 427-489.

Андрей Валерьевич Кудинов (Andrey V. Kudinov)

Институт проблем передачи информации им. А. А. Харкевича Российской академии наук

E-mail: kudinov@iitp.ru

\section{Илья Борисович Шапировский}

(Ilya B. Shapirovsky)

Институт проблем передачи информации

им. А. А. Харкевича Российской академии наук

E-mail: shapir@ittp.ru
Представлено С. К. Ландо Принято редколлегией 23.06.2015 